\title{
Avaliação da intervenção cognitivo- comportamental em gestão do stress em pacientes com fadiga oncológica, em radioterapia
}

Evaluation of cognitive behavioral intervention on stress management in patients suffering from cancer-fatigue in radiotherapy

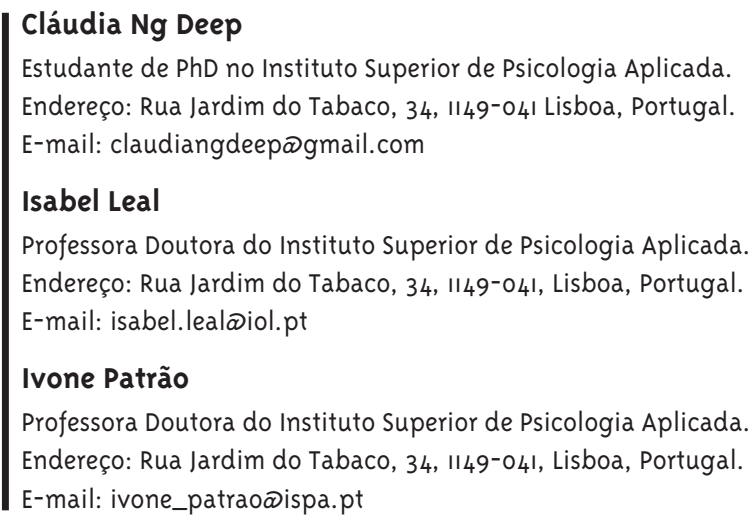

Estudante de PhD no Instituto Superior de Psicologia Aplicada. Endereço: Rua Jardim do Tabaco, 34, 1149-041 Lisboa, Portugal. E-mail: claudiangdeepœgmail.com

Isabel Leal

Professora Doutora do Instituto Superior de Psicologia Aplicada. Endereço: Rua Jardim do Tabaco, 34, 1149-041, Lisboa, Portugal. E-mail: isabel.lealळiol.pt

Ivone Patrão

Professora Doutora do Instituto Superior de Psicologia Aplicada. Endereço: Rua Jardim do Tabaco, 34, 1149-041, Lisboa, Portugal.

E-mail: ivone_patrao®ispa.pt

\section{Resumo}

Apresentam-se os resultados relativos a padrões de regulação emocional, percepção e satisfação com o suporte social após intervenção cognitivo-comportamental em gestão do stress (IGSCC) em pacientes em radioterapia com fadiga oncológica. Compara-se o grupo de controlo ( $n=35$ radioterapia sem IGSCC) e o grupo experimental $(n=35$ radioterapia com IGSCC) no início e fim do tratamento tendo em conta variáveis sociodemográficas, Escala de ansiedade, depressão e stress, Escala de perceção e satisfação com o suporte social e o Termómetro da emoção. No grupo experimental, após IGSCC, há descida significativa ( $p<.001)$ da ansiedade, depressão e stress e aumento da percepção e satisfação com o suporte social. No grupo de controlo há subida significativa ( $p<.001)$ da desregulação emocional (ansiedade, depressão e stress) e subida da insatisfação com o suporte social. Salienta-se a eficácia da IGSCC sobre estados emocionais e percepção e satisfação com o suporte social em pacientes em radioterapia e com fadiga oncológica.

Palavras-chave: Fadiga-oncológica; Suporte-social; Stress; Ansiedade; Depressão; Cognitivo-comportamental; Radioterapia. 


\section{Abstract}

This article presents the results for patterns of emotional regulation, perception and satisfaction with social support after a Cognitive-behavioral stress management intervention (IGSCC) in patients with oncologic fatigue, undergoing radiation therapy. A comparison was established between the control group ( $\mathrm{n}=35$ without radiotherapy, IGSCC) and the experimental group ( $\mathrm{n}=35$ radiotherapy, IGSCC) at the beginning and end of treatment, considering socio-demographic variables; the Anxiety, Depression and Stress Scale; the Perception and Satisfaction with Social Support Scale and the Emotion Thermometer. In the experimental group, after the IGSCC, there was a significant decrease $(\mathrm{p}<$.oo1) in anxiety, depression and stress and an increase in the perception and satisfaction with social support. In the control group, there is a significant increase $(p<.001)$ in emotional deregulation (anxiety, depression and stress) and dissatisfaction with social support. We highlight the effectiveness of IGSCC on emotional states, perception and satisfaction with social support in patients with oncologic fatigue undergoing radiation therapy.

Keywords: Cancer-Related-Fatigue; Social-Support; Stress; Anxiety; Depression; Cognitive-Behavioral; Radiotherapy.

\section{Introdução}

A literatura da especialidade (Antoni e Lutgendorf, 2007; Brix e col., 2008; Dhruva e col., 2010; Matthews e Cook, 2009; Schneider e Lopes, 2004) refere que certos pacientes oncológicos apresentam desordens como ansiedade, depressão, stress associadas a baixa percepção e satisfação com o suporte social. É a fadiga oncológica, cuja sintomatologia tende a aumentar durante os tratamentos como a radioterapia: quando se espera o tratamento, quando neste há uma alteração, quando falha ou no seu final. 0 mal-estar presente origina enfrentamento inadequado e baixa adesão ao tratamento, aumento do tempo de hospitalização, alta percepção da dor e redução da capacidade de resposta, aumento do risco de recaída e de suicídio. Ainda Schneider e Lopes (2004) relacionam a fadiga oncológica com a doença e o tratamento e caracterizam-na como diferente da fadiga em geral: é persistente, interfere no dia a dia do paciente, é marcada pela falta de energia, pela sonolência, fraqueza muscular, perturbação do humor, com diminuição da performance cognitiva (ex: concentração) e da qualidade de vida, e com custos económicos acrescidos. Sendo que persiste muito tempo, após o fim dos tratamentos. No entanto esse efeito colateral e multifactorial raramente é abordado em cenário clínico, constituindo-se um problema prolongado, desnecessariamente, no tempo.

Kim e colaboradores (2009) procuraram perceber a severidade sintomatológica da fadiga oncológica, no final da radioterapia, e verificaram que a ansiedade associada aliava-se à falta de energia, dificuldade em dormir, sonolência e suores constantes. Esta ansiedade, tal como sintomas depressivos, é preditora desta fadiga (Vries e col., 2008) e compromete a qualidade de vida em ambulatório (Decker, e col., 2006). Complementarmente há literatura da especialidade que defende que a relaxação durante a radioterapia, com componente educacional, reduz a tensão, a depressão, a ira e a fadiga (Decker e col., 20o6): defende-se uma intervenção cognitivo-comportamental em gestão do stress na redução da ansiedade e seus pensamentos intrusivos com resultados durante o tratamento e após um ano (Antoni e Lutgendorf, 2007; Gottlieb eWachala, 2006) e a expressão emocional capaz de incrementar a percepção de controlo, 
o bem-estar emocional, um coping/enfrentamento eficaz, a diminuição do medo de recorrência e a preocupação com o cancro e a ansiedade (Cameron e col., 2006). Quanto ao stress, sabe-se que o seu processo fisiológico estimula os tumores: hormonas como as catecolaminas e gluco-corticoides surgem durante eventos de vida estressantes e podem acelerar o crescimento tumoral, modelar a sua dinâmica e aumentar o risco de alterações pré-cancerígenas, sendo pertinente ajudar os pacientes a gerir os factores estressantes e a diminuir o distress de forma a contribuir para um controlo neuro-endócrino necessário ao controlo da doença oncológica (Antoni e Lutgendorf, 2007). Nunes e colaboradores (2007) referiram que o tratamento ao cancro é acompanhado por significante stress psicológico e que a relaxação com visualização é efetiva na sua redução, tal como é na ansiedade e depressão. E Krischer e colaboradores (2007) salientam que pacientes em radioterapia e com níveis elevados de stress apresentam maior qualidade de vida se forem sujeitos a treino de autogestão do stress. Ainda Purcell e colaboradores (2009) acrescentaram que o aumento da fadiga oncológica está associado a um nível de menor performance, à depressão e problemas do sono. E Dhruva e colaboradores (2010) verificaram que há uma clara relação entre fadiga oncológica e depressão, baixo índice de massa corporal, perturbação do sono, ansiedade e morbilidade médica. 0 humor depressivo constitui-se como bom preditor dessa fadiga antes, durante e após o tratamento e as intervenções que visam reduzi-lo parecem ser eficazes (Von Ah e col., 2008).

Quanto à percepção e satisfação com o suporte social, a literatura defende que os pacientes mais susceptíveis à fadiga oncológica são mulheres com necessidade de suporte psicossocial (Brix e col., 2008), e que há correlação negativa entre esta necessidade e a qualidade de vida e resiliência, um bom preditor da fadiga (Brix e col., 2008). Antoni e Lutgendorf (2007) referem que a baixa percepção de suporte social leva à progressão da doença pois substâncias neuroendócrinas (catecolaminas e cortisol) desregulam a resposta imunológica ao cancro. Mas o optimismo e o suporte social permitem maior sobrevivência por contribuírem para parâmetros imunológicos mais adequados. Matthews e Cook
(2009) concordam que a percepção de suporte social e o optimismo, durante a radioterapia, influencia o impacto do diagnóstico e tratamento e acrescentam que o rastreio do optimismo permite identificar as mulheres em fadiga oncológica, sendo necessário investigar intervenções que promovam o ajustamento psicológico durante a trajetória oncológica. Esses pacientes procuram suporte social de forma insuficiente e avaliam a rede social (família, amigos e organismos sociais) como insatisfatória, mas a terapia cognitivo-comportamental facilita o enfrentamento da doença (por meio das experiências em atividades que suportem as práticas e habilidades de enfrentamento e expressão das emoções e afectividades), e pode melhorar a percepção de apoio social, sendo pertinente também fortalecer a rede social desses pacientes (Deep e col., 2012).

Segundo Antoni e Lutgendorf (2007), as intervenções psicossociais que reduzem a tensão física e a ansiedade (relaxação), que alteram o humor negativo (por exemplo, através da reestruturação cognitiva), que ajudam a incrementar a capacidade de buscar e receber suporte social e a expressar sentimentos, através de treinos de assertividade e expressão da ira (Deep, 2010) podem modelar a atividade hormonal e favorecer o processo de recuperação da doença. Ainda segundo Antoni e Lutgendorf (2007), as intervenções que combinam técnicas cognitivo-comportamentais com relaxamento potencializam o funcionamento imunológico durante a radioterapia, modificam a desregulação neuroendócrina e têm implicações na sobrevivência dos pacientes. Assim, coloca-se como questão de investigação perceber qual o impacto da relaxação muscular, da reestruturação cognitiva e do treino em estratégias de enfrentamento (IGSCC) sobre os padrões de regulação emocional e sobre a perceção e satisfação com o suporte social, comparando ambos os grupos, antes e após o tratamento.

\section{Método}

O grupo experimental (GE) é composto por 35 sujeitos com fadiga oncológica no início da radioterapia, predominantemente do sexo feminino com idades entre 32 e 80 anos (média $=60,54$ anos). 0 grupo de controlo (GC) é composto por 35 sujeitos 
com fadiga oncológica no início da radioterapia, predominantemente do sexo feminino com idades entre 40 e 86 anos (média $=60,83$ ). Em ambas as amostras, a maioria é casada, caucasiana, com escolaridade básica. Avalia o ambiente familiar como favorável, com estabilidade socioeconómica (não estando empregada por estar em idade de reforma ou pré-reforma). Ambas as amostras referem estar a tomar medicação psiquiátrica e/ou outro tipo de medicação com regularidade e estar em radioterapia curativa, sendo o tumor atual mais presente o da mama, seguindo-se o do sistema genital e urinário. Referem que sendo esta a $1^{a}$ patologia oncológica, já sofreram outras doenças físicas graves vivenciadas "nervosamente" ou "muito nervosamente".

Quanto aos instrumentos, recorreu-se a um Questionário de Caracterização Sociodemográfica construído para o efeito e composto por itens que avaliam características sociais, demográficas e clínicas, tendo por base a revisão da literatura: idade, estado civil, escolaridade, género, raça/etnia, ambiente familiar e situação económica, empregabilidade, medicação atual, tipo de radioterapia, tipo e localização do tumor atual, doenças oncológicas anteriores, outras doenças anteriores e como foram emocionalmente vivenciadas. Recorreu-se ao Termómetro Emocional - TE composto por sete itens que avaliam níveis de sofrimento emocional, ansiedade, depressão, revolta, impacto e duração dos sintomas bem como o nível de ajuda que a pessoa percebe necessitar. Este instrumento foi validado para a população portuguesa oncológica adulta (Bizarro e col., 2012) e o paciente indica o quanto tem sentido na semana anterior e no próprio dia numa escala de onze níveis, sendo que, a partir do nível quatro, considera-se que a pessoa está sob influência de algum quadro patológico capaz de afetar o seu bem-estar. Quanto mais elevado o nível indicado, maior o sofrimento psíquico sentido. A consistência interna é adequada ( $\alpha=0.91$ ) e os sete fatores são descritos na literatura da especialidade como presentes em situações de fadiga oncológica: recorreu-se a este instrumento como forma de detectar indivíduos em situação de fadiga oncológica e suscetíveis de participar no estudo. Ainda usou-se a Escala de Ansiedade, Depressão e Stress (Eads 21) validada por Pais-Ribeiro e colaboradores (2004) que avalia ansiedade, depressão e stress em sete itens (em cada subescala) que remetem para sintomas emocionais negativos experienciados na última semana, com quatro possibilidades de resposta (Lovibond e Lovibond, 1995). A consistência interna é adequada (depressão- $\alpha=0.85$; ansiedade- $\alpha=0.74$; stress $-\alpha=0.81$ ). Em cada subescala, obtém-se pontuação pela soma dos resultados dos itens sendo a mínima zero e a máxima vinte e um: quanto mais elevada a pontuação, maior o estado afetivo negativo. A ansiedade remete para a excitação do sistema autónomo, ansiedade situacional e experiências subjetivas, incluindo: excitação sistema autónomo; efeitos músculo esqueléticos; ansiedade situacional e experiências subjetivas de ansiedade. A depressão compreende sentimentos de desânimo e desvalorização da vida, autodepreciação e desmotivação, perda da capacidade de sentir prazer, inércia, disforia, falta de interesse/envolvimento. O stress abrange a dificuldade em relaxar, irritabilidade, tensão nervosa e agitação. Por último usou-se a Escala de Percepção e Satisfação com o Suporte Social (ESSS) validada por Pais-Ribeiro (1999); avalia o suporte social percebido e grau de satisfação inerente, em 15 itens, sendo o $1^{\circ}$ - factor a satisfação com as amizades (itens 1, 2, 3, 4, 5 medem o grau de satisfação com os amigos), o segundo factor a intimidade (itens 6, 7, 8, 9 medem a percepção da existência de suporte social íntimo), o terceiro factor a satisfação com a família (itens 10, 11, 12 medem a satisfação com o suporte social familiar existente) e o quarto factor as atividades sociais (itens 13, 14, 15 medem a satisfação com as atividades sociais que realiza). Numa escala com cinco posições de resposta, o sujeito assinala a sua resposta. A consistência interna é adequada $(\alpha=0.85)$ e o factor que melhor explica o resultado total da escala é o primeiro.

Quanto aos procedimentos, após pesquisa bibliográfica, solicitou-se autorização às entidades envolvidas e mediante consentimento informado, os pacientes foram sujeitos ao TE. Os que apresentaram valores concordantes com um estado de fadiga oncológica $(>4)$ foram, aleatoriamente, divididos pelo GC e GE e sujeitos aos restantes instrumentos de avaliação antes de iniciarem o tratamento (GC1 e GE1) e após o tratamento (GC2 e GE2). 0 GC foi apenas sujeito a tratamento convencional 
de radioterapia e o GE foi sujeito ao tratamento por radioterapia com IGSCC (relaxação muscular, reestruturação cognitiva e treino em estratégias de enfrentamento). Como variáveis, salienta-se stress, ansiedade, depressão, satisfação com as amizades, intimidade, satisfação com a família, atividades sociais. A análise estatística decorreu com base no Statistical Package for Social Sciences - SPSS (v.19, SPSS Inc., Chicago, IL) e em cada uma das sete variáveis em estudo, verificou-se se o GC1 e o GE1 são inicialmente grupos homogéneos/ comparáveis. Posteriormente, no GE avaliou-se o impacto da IGSCC sobre cada variável analisando-se e comparando-se os resultados obtidos antes e após a radioterapia com IGSCC. Em seguida analisou-se o impacto do tratamento convencional sem IGSCC, no GC, sobre cada variável e para finalizar avaliou-se as diferenças entre GE2 e GC2 de forma a verificar se as diferenças entre o fim de ambos os tratamentos eram ou não significativas. Como tal procedeu-se à estatística descritiva de cada variável e o teste de Kolmogorov-Smirnov permitiu verificar o tipo de distribuição amostral: quando normal recorreu-se ao teste-T para amostras emparelhadas (sendo p>0.05 sinónimo de distribuição normal) e quando anormal recorreu-se ao teste de Wilcoxon para amostras emparelhadas, considerando-se valores $\mathrm{p}<0.05$ indicativos de diferenças significativas. Ainda na escala de Percepção e Satisfação com o Suporte Social, recodificou-se os itens $2,3,4,5,7,8,10,11,12$, pois devem ser cotados invertidamente.

\section{Apresentação e Análise dos Resultados}

Ao analisar os dados referentes ao Termómetro Emocional (Quadro 1), verifica-se que no GC e GE encontram-se valores médios (numa escala de onze pontos) sinónimos de mal-estar emocional e clara fadiga oncológica. Comprova-se que em ambos os grupos os sujeitos são adequados à participação neste estudo.

Quanto aos padrões de regulação emocional (Eads-21), os valores alpha de Cronbach mostram-se satisfatórios como sinónimo de homogeneidade: GC1 (.941); GC2 (.935); GE1 (.899); GE2 (.894). Quadro I - Termómetro Emocional - GC $(N=35)$ e GE
$(N=35)$, Portugal, 2012

\begin{tabular}{|l|c|c|}
\hline Termómetro Emocional & GC $(\dot{x})$ & GE $(\dot{x})$ \\
\hline Sofrimento Emocional & 7.29 & 7.97 \\
\hline Ansiedade & 7.46 & 7.80 \\
\hline Depressão & 7.17 & 7.71 \\
\hline Revolta & 5.71 & 5.57 \\
\hline Duração do Estado Emocional & 6.97 & 6.71 \\
\hline Impacto & 7.69 & 8.34 \\
\hline Necessidade de Ajuda & 6.14 & 7.11 \\
\hline
\end{tabular}

CG - Grupo de Controlo/ GE - Grupo Experimental.

0 quadro 2 apresenta os resultados relativos à ansiedade, depressão e stress. Verifica-se que quanto aos valores médios, no GC todos os parâmetros subiram. No GE após a IGSCC encontram-se valores médios menores que quatro sugerindo equilíbrio psicoemocional, sendo que todos os três parâmetros desceram.

Efetivamente, numa análise mais aprofundada, verifica-se que no GC para a ansiedade ( $T=-5.1$; $\mathrm{p}<.001)$, a depressão ( $\mathrm{T}=-4.5 ; \mathrm{p}<.001)$ e o stress ( $\mathrm{T}=-$ 6.o; $\mathrm{p}<.001$ ) existem diferenças significativas entre antes e após o tratamento, sendo que no segundo momento há subida significativa de todos os parâmetros como sinónimo do aumento do mal-estar emocional após tratamento por radioterapia. Ainda para o GE verifica-se que para a ansiedade $(\mathrm{T}=10.66$; $\mathrm{p}<.001)$, a depressão $(\mathrm{Z}=-5.16$; $\mathrm{p}<.001)$ e o stress $(\mathrm{Z}=-$ 5.089; $\mathrm{p}<.001)$ existem diferenças significativas entre antes e após o tratamento com IGSCC, sendo que no segundo momento há descida significativa de todos os parâmetros como sinónimo da diminuição do mal-estar emocional no GE após tratamento por radioterapia com IGSCC.

Quanto à percepção e satisfação com o suporte social, os valores alpha de Cronbach mostram-se satisfatórios como sinónimo de homogeneidade: GC1 (.892); GC2 (.6o6); GE1 (.751); GE2 (.776). O quadro 3 apresenta a estatística descritiva da percepção e satisfação com o suporte social (família, amigos e organismos sociais). 
Quadro 2 - Estatística Descritiva Eads2। - GCl e 2/ GEI e 2 ( $N=35$ ), Portugal, 2012

\begin{tabular}{|l|c|c|c|c|}
\hline Padrões Emocionais & Média & Desvio Padrão & Mínimo & Máximo \\
\hline GCIAnsiedade & 9.28 & 4.793 & .00 & 22.00 \\
\hline GC2Ansiedade & 13.45 & 4.118 & 6.00 & 23.00 \\
\hline GCIDepressão & 11.65 & 5.609 & .00 & 21.00 \\
\hline GC2Depressão & 15.34 & 4.595 & 5.00 & 22.00 \\
\hline GCIStress & 12.20 & 4.457 & .00 & 20.00 \\
\hline GC2Stress & 16.45 & 4.258 & 7.00 & 27.00 \\
\hline GEIAnsiedade & 9.97 & 3.988 & .00 & 18.00 \\
\hline GE2Ansiedade & 1.48 & 2.267 & 3.00 & 10.00 \\
\hline GEIDepressão & 13.80 & 5.138 & .00 & 20.00 \\
\hline GE2Depressão & 1.71 & 2.308 & 6.00 & 7.00 \\
\hline GEIStress & 14.08 & 4.279 & .00 & 21.00 \\
\hline GE2Stress & 2.42 & 2.862 & & 8.00 \\
\hline
\end{tabular}

Quadro 3 - Estatística Descritiva ESSS - GCI e 2 / GE I e 2 ( $N=35$ ), Portugal, 2012

\begin{tabular}{|c|c|c|c|c|}
\hline Percepção e Satisfação com o Suporte Social & Média & Desvio Padrão & Mínimo & Máximo \\
\hline GCISatisfação Amizades & 13.11 & 4.574 & 5.00 & 24.00 \\
\hline GC2Satisfação Amizades & 10.31 & 1.859 & 5.00 & 13.00 \\
\hline GEıSatisfação Amizades & 10.28 & 1.903 & 6.00 & 15.00 \\
\hline GE2Satisfação Amizades & 20.42 & 2.200 & 15.00 & 25.00 \\
\hline GCI Intimidade & 9.57 & 2.953 & 4.00 & 16.00 \\
\hline GC2 Intimidade & 7.80 & 1.323 & 4.00 & 10.00 \\
\hline GEI Intimidade & 7.68 & 2.011 & 4.00 & 14.00 \\
\hline GE2 Intimidade & 15.60 & 1.479 & 12.00 & 18.00 \\
\hline GCı Satisfação Família & 8.68 & 3.570 & 3.00 & 15.00 \\
\hline GC2 Satisfação Família & 6.17 & 1.316 & 3.00 & 9.00 \\
\hline GEı Satisfação Família & 5.91 & 1.771 & 3.00 & 12.00 \\
\hline GE2 Satisfação Família & 12.74 & 1.421 & 9.00 & 15.00 \\
\hline GCI Satisf. Atividades Sociais & 8.62 & 2.690 & 3.00 & 15.00 \\
\hline GC2 Satisf. Atividades Sociais & 8.25 & 1.120 & 6.00 & 11.00 \\
\hline GEI Satisf. Atividades Sociais & 7.80 & 1.183 & 6.00 & 10.00 \\
\hline GE2 Satisf. Atividades Sociais & 11.40 & 1.005 & 9.00 & 13.00 \\
\hline
\end{tabular}

No quadro 3 verifica-se que no GC, entre o primeiro e o segundo momento de avaliação a satisfação com as amizades desceu significativamente de um valor médio igual a 13.11 para $10.31(\mathrm{~T}=4.0$; $\mathrm{p}<.001$ ), a intimidade também desceu significativamente de um valor médio igual a 9.57 para 7.80 $(\mathrm{T}=3.9 ; \mathrm{p}<.001)$ e a satisfação com as atividades sociais desceu (sem diferenças significativas) de um valor médio igual a 8.62 para um valor médio igual a $8.25(\mathrm{~T}=.92 ; \mathrm{p}=.362)$. Ainda no quadro 3 verifica-se que quanto à satisfação com a família o valor médio de 8.68 desceu significativamente para 6.17 $(\mathrm{Z}=-3.48 ; \mathrm{p}<.001)$.

Quanto ao GE, o quadro 3 revela que a satisfação com as amizades subiu significativamente ( $\mathrm{T}=-19.1$; $\mathrm{p}<.001)$ tal como a intimidade $(\mathrm{T}=-22.0 ; \mathrm{p}<.001)$, a satisfação com a família ( $Z=-5.18$; $p<.001)$ e a satisfação com as atividades sociais $(\mathrm{Z}=-5.04 ; \mathrm{p}<.001)$. 


\section{Discussão dos resultados}

Esta investigação surge no contexto de dados empíricos contraditórios quanto à eficácia da intervenção em gestão do stress cognitivo-comportamental em pacientes com fadiga oncológica e sujeitos à radioterapia (Goedendorp e col., 2009). Efetivamente a comunidade científica tem apresentado algumas dúvidas quanto à eficácia das intervenções cognitivo-comportamentais sobre a gestão da fadiga oncológica: alguns estudos referem resultados positivos enquanto outras investigações mostram-se inconclusivas quanto à eficácia deste tipo de intervenção. Assim, foi também objectivo deste estudo avaliar o impacto da IGSCC sobre padrões de regulação emocional e perceção e satisfação com o suporte social nestes pacientes.

Os resultados obtidos vão na mesma direcção que os obtidos por Yoo e colaboradores (2009). Estes investigadores estudaram os efeitos de uma intervenção cognitivo-comportamental sobre a ansiedade e a depressão em mulheres com cancro da mama em tratamento por radioterapia (com aconselhamento, educação sobre tratamentos, estratégias para enfrentar a radioterapia e exercícios de reabilitação) e concluíram que após a intervenção, o GE apresentou níveis muito mais baixos de ansiedade e depressão, comparando com o GC.

Neste estudo também se verificou uma diminuição significativa da ansiedade, stress e depressão após tratamento com IGSCC (relaxação muscular, reestruturação cognitiva e treino em estratégias de enfrentamento), enquanto no GC assistiu-se a um aumento significativo destes parâmetros $(p<.001)$ após tratamento convencional. Quanto à percepção e satisfação com o suporte social (ou seja a percepção de que há pessoas que se preocupam e disponibilizam para estar próximas de quem se pode confiar, aumentando a satisfação do paciente) os resultados são também conclusivos e complementares aos obtidos nos padrões de regulação emocional pois em geral a satisfação com as amizades, a percepção e satisfação com a intimidade, a satisfação com as relações familiares e a satisfação com as atividades sociais aumentam no GE e diminuem no GC ao longo do tratamento por radioterapia. É importante salientar que a subescala da satisfação com os amigos e as amizades é a que melhor explica a satisfação com o suporte social em termos gerais, e no GC assiste-se a uma diminuição significativa de tal satisfação ao longo do tratamento por radioterapia enquanto no grupo experimental assiste-se a uma subida significativa de tal satisfação. Estes resultados vão na mesma direcção que os obtidos por Santana e colaboradores (2008) quando afirmam a necessidade de fortalecer a rede social destes pacientes e melhorar a percepção de apoio social (de forma a promover a saúde) através de técnicas como treino em competências sociais, expressão e regulação das emoções. Um dos conteúdos abordados nesta intervenção foi o treino em assertividade (Deep, 2010) com resultados positivos ao nível da capacidade de auto afirmação e expressão emocional. Reconhecendo-se que o suporte social não depende apenas do tamanho da rede social, mas também do seu conteúdo (Moorey, 2010; Brothers e col., 2010) e tal como salientou Matthews e Cook (2009) está relacionado com maior optimismo e bem-estar. Os resultados ainda vão na mesma direcção que os obtidos por Brix e colaboradores (2008) e exaltam a eficácia da intervenção cognitivo-comportamental que acompanha e complementa a radioterapia. Sendo que a presença de suporte social ( percebida pelo paciente e que o satisfaça) contribui para baixos níveis de fadiga oncológica e aumento da sua qualidade de vida. Assim, verifica-se que doentes oncológicos com fadiga oncológica (em estado de sofrimento emocional e físico, com padrões de ansiedade, stress e depressão, com níveis altos de revolta e clara necessidade de ajuda) quando são sujeitos a mais um tratamento como a radioterapia tendem a sofrer um aumento do mal-estar já instalado com maior insatisfação diante de amizades, relações familiares, atividades sociais em geral, bem como tendem a experimentar menor intimidade e menor satisfação com tal. Fala-se de isolamento e mal-estar social. Mas estes doentes com fadiga oncológica se forem sujeitos a mais um tratamento, como a radioterapia, mas se estiverem convenientemente acompanhados do ponto de vista psicológico já tenderão a apresentar uma subida do bem-estar emocional com maior percepção e satisfação com o suporte social. 


\section{Conclusão}

Conclui-se tal como Jacobsen (2008) que os diversos dados científicos têm comprovado a importância do acompanhamento psicossocial na redução do mal-estar emocional no doente oncológico, pelo que se torna imprescindível levar estes dados em linha de conta quando se trata estes doentes. É um grande desafio persuadir os técnicos a adoptar estratégias e intervenções mais adequadas. Com este artigo pretende-se sublinhar como é pertinente usar as evidências científicas para promover mudança na prática profissional de forma a Psico-Oncologia atingir o seu potencial no tratamento de pessoas com cancro. Sublinha-se o pertinente impacto da gestão do stress oncológico em pacientes com fadiga oncológica através da aprendizagem e prática da relaxação, da reestruturação cognitiva e da aprendizagem de estratégias de enfrentamento adequadas às necessidades individuais de cada paciente.

\section{Referências}

ANTONI, M.; LUTGENDORF, S. Psychosocial factors and disease progression in cancer. Current Directions in Psychological Science, Miami, v. 16, n. 1, p. 42-46, 2007.

BIZARRO, J.; PATRÃO, I.; DEEP, C. Contributo para a validação do TE (Termómetro Emocional) numa amostra portuguesa com diagnóstico de cancro. In: PAIS-RIBEIRO, J. L. et al. Desafios à promoção da saúde em doenças crónicas: atas do $9^{\circ}$ Congresso Nacional de Psicologia da Saúde. Lisboa: Placebo, 2012. p. 152-159.

BRIX, C. et al. The need for psychosocial support and its determinants in a sample of patients undergoing radiooncological treatment of cancer. Journal of Psychosomatic Research, Manchester, v. 65, n. 6, p. 541-548, 2008.

BROTHERS, B.; THORNTON, L.; ANDERSEN, B. Cognitive and behavioral interventions. In: HOLLAND, J. et al. Psycho-oncology. Oxford: Oxford University Press, 2010. p. 415-421.
CAMERON, L. et al. Changes in emotion regulation and psychological adjustment following of a group psychosocial support program for women recently diagnosed with breast cancer. Psycho-Oncology Journal, Charlottesville, v. 16, n. 3, p. 171-18o, 2006.

DECKER, T. W.; CLINE-ELSEN, J.; GALLAGHER, M. Relaxation therapy as an adjunct in radiation oncology. Journal of Clinical Psychology, Chichester, v. 48, n. 3, p. 388-393, 2006.

DEEP, C. Sal e luz: viver em assertividade. Lisboa: Paulus, 2010.

DEEP, C.; LEAL, I.; PATRÃO, I. Avaliação do impacto de uma intervenção cognitivocomportamental em gestão do stress sobre estados emocionais e crenças, em pacientes com fadiga oncológica sujeitos a radioterapia - dados preliminares. In: PAIS-RIBEIRO, J. L. et al. Desafios à promoção da saúde em doenças crónicas: atas do $9^{\circ}$ Congresso Nacional de Psicologia da Saúde. Lisboa: Placebo, 2012. p. 348354 .

DHRUVA, A. et al. Trajectories of fatigue in patients with breast cancer before, during and after radiation therapy. Cancer Nursing Journal, Washington, DC, v. 33, n. 3, p. 201-212, 2010.

GOEDENDORP, M. et al. Psychosocial interventions for reducing fatigue during cancer treatment in adults. Cochrane Database of Systematic Reviews, London, v. 21, n. 1, Não paginado, 2009.

GOTTLIEB, B.; WACHALA, E. Cancer support groups: a critical review of empirical studies. Psycho-Oncology Journal, Charlottesville, v. 15, n. 5, p. 379-400, 2006.

JACOBSEN, P. Promoting evidence-based psychosocial care for cancer patients. PsychoOncology Journal, Charlottesville, v. 18, n. 1, p. 6-13, 2008.

KIM, E. et al. Differences in symptom clusters identified using occurrence rates versus symptom severity ratings in patients at the end of radiation therapy. Cancer Nursing Journal, Washington, DC, v. 32, n. 6, p. 429-436, 2009. 
KRISCHER, M. et al. Self-administered stress management training in patients undergoing radiotherapy. Journal of Clinical Oncology, Alexandria, v. 25, n. 29, p. 4657-4662, 2007.

LOVIBOND, P.; LOVIBOND, S. The structure of negative emotional states: comparison of the Depression Anxiety Stress Scales (DASS) with the Beck Depression and Anxiety Inventories. Behaviour Research and Therapy, Chapel Hill, v. 33, n. 3, p. 335-343, 1995.

MATTHEWS, E.; COOK, P. Relationship among optimism, well-being, self-transcendence, coping and social support in women during treatment for breast cancer. Psycho-Oncology Journal, Charlottesville, v. 18, n. 7, p. 716-726, 2009.

MOOREY, S. Cognitive therapy. In: HOLLAND, J. et al. Psycho-oncology. Oxford: Oxford University Press, 2010. p. 402-407.

NUNES, D. et al. Relaxation and guided imagery program in patients with breast cancer undergoing radiotherapy is not associated with neuroimmunomodulatory effects. Journal of Psychosomatic Research, Manchester, v. 63, n. 6, p. 647-655, 2007.

PAIS-RIBEIRO, J. Escala de Satisfação com o Suporte Social (ESSS). Análise Psicológica, Lisboa, v. 3, n. 17, p. 547-558, 1999.

Recebido em: 28/05/2012 Reapresentado em: 07/08/2013 Aprovado em: 26/09/2013
PAIS-RIBEIRO, J.; HONRADO, A.; LEAL, I. Contribuição para o estudo da adaptação portuguesa das Escalas de Ansiedade, Depressão e Stress (EADS) de 21 itens de Lovibond e Lovibond. Psicologia: Saúde e Doenças, Lisboa, v. 5, n. 2, p. 229-239, 2004.

PURCELL, A. et al. A multidimensional examination of correlates of fatigue during radiotherapy. Cancer Journal, New Haven, v. 116, n. 2, p. 529-537, 2009.

SANTANA, J.; ZANIN, C.; MANIGLIA, J. Pacientes com câncer: enfrentamento, rede social e apoio social. Paidéia, São José do Rio Preto, v. 18, n. 40, p. 371-384, 2008.

SCHNEIDER, I.; LOPES, S. Fadiga relacionada ao câncer. Pratica Hospitalar, São Paulo, v. 6, n. 35, Não paginado, 2003.

VON AH, D.; KANG, D.; CARPENTER, J. Predictors of cancer-related fatigue in women with breast cancer before, during, and after adjuvant therapy. Cancer Nursing Journal, Washington, DC, v. 31, n. 2, p. 134-144, 2008.

VRIES, J.; STEEG, A.; ROUKEMA, J. Determinants of fatigue 6 and 12 month after surgery in women with early-stage breast cancer: a comparison with benign breast problems. Journal of Psychosomatic Research, Tilburg, v. 66, n. 6, p. 495-502, 2008.

YOO, M.; LEE, H.; YOON, J. Effects of a cognitivebehavioral nursing intervention on anxiety and depression in women with breast cancer undergoing radiotherapy. Journal Korean Academic Nurses, Busan, v. 39, n. 2, p. 157-165, 2009. 